\title{
Fault diagnosis based on dial-test data in datacenter networks
}

\author{
QI Xiaogang ${ }^{1,3}$, WANG Bingchun ${ }^{1, *}$, and LIU Lifang ${ }^{2,3}$ \\ 1. School of Mathematics and Statistics, Xidian University, Xi' an 710071, China; \\ 2. School of Computer Science and Technology, Xidian University, Xi' an 710071, China; \\ 3. Xidian-Ningbo Information Technology Institute, Ningbo 315200, China
}

\begin{abstract}
The fast growth of datacenter networks, in terms of both scale and structural complexity, has led to an increase of network failure and hence brings new challenges to network management systems. As network failure such as node failure is inevitable, how to find fault detection and diagnosis approaches that can effectively restore the network communication function and reduce the loss due to failure has been recognized as an important research problem in both academia and industry. This research focuses on exploring issues of node failure, and presents a proactive fault diagnosis algorithm called heuristic breadth-first detection (HBFD), through dynamically searching the spanning tree, analyzing the dial-test data and choosing a reasonable threshold to locate fault nodes. Both theoretical analysis and simulation results demonstrate that HBFD can diagnose node failures effectively, and take a smaller number of detection and a lower false rate without sacrificing accuracy.
\end{abstract}

Keywords: datacenter network, node failure, proactive fault diagnosis.

DOI: $10.21629 / J S E E .2019 .05 .19$

\section{Introduction}

As the advent of big data era, increasing demand of cloud computing has caused larger scale of data center networks. Nowadays, data center network contains hundreds of thousands of servers connected by network interface cards (NICs), switches and routers, cables and fibers [1], and most of these servers are distributed and have a high traffic. The datacenter network has three main topologies: fat tree topology, FiCoon topology and DCell topology. In such large systems, detecting and locating faults is very important for network management systems to restore network communication by specific failure recovery mechanism.

Manuscript received March 20, 2018

*Corresponding author.

This work was supported by the National Natural Science Foundation of China (61877067; 61572435), the joint fund project of the Ministry of Education - the China Mobile (MCM20170103), Xi' an Science and Technology Innovation Project (201805029YD7CG13-6), and Ningbo Natural Science Foundation (2016A610035; 2017A610119).
Although many efforts have been devoted to studying the fault diagnosis strategy, research on fault diagnosis in datacenter networks is still relatively rare, and taking into account the features of large-scale, flexible topologies, highspeed broadband and efficient network protocols of datacenter network [2], there are still many problems to be addressed.

(i) Diagnosis complexity. Besides causing higher temporal and spatial complexity for fault locating, the increasing network size also results in more sophisticated fault diagnosis due to the dynamic, incomplete and uncertain information. Thus, it is challenging to design an effective algorithm to reduce the complexity of the fault diagnosis.

(ii) The increase of control overhead. Datacenters can dramatically reduce the algorithm execution time, but it may also lead to the increasing controller overhead. One possible solution is to apply a participation strategy to controling the number of monitors, while another strategy is to improve the effectiveness of detective data to reduce the data amount.

(iii) Network uncertainty. When the network has large traffic or some malicious nodes, it increases the probabilities of the packet loss, the transmission latency and the number of error symptoms. All of them will cause incomplete and inaccurate information, and then increase the difficulty of fault location for management systems. Thus, how to quantify and reduce the uncertainty of the network is also valuable for fault diagnosis.

Within the computer science community, a growing body of work has focused on fault diagnosis in communication networks. The existing research of fault diagnosis can be classified into three categories: passive monitoring approaches, active probing techniques and the method based on network logs.

(i) Passive monitoring approaches

Passive monitoring techniques use monitoring agents to detect network conditions. The information used to 
infer components status is obtained passively, and it also has been proved that locating fault nodes are nondeterministic polynomial (NP)-hard [3]. Many researchers have focused on decreasing the complexity of testing [4,5]. However, most of these approaches assume that networks are low-bandwidth and low traffic overhead [6]. Model-based fault diagnosis also has attracted the attention of many scholars [7,8], but these models may be difficult to obtain or adapt to the flexible topology of datacenter networks.

(ii) Active probing techniques

Proactive fault diagnosis has attracted many scholars' attention in recent years. The method typically uses probes to detect the condition of servers. These selected probes are transmitted to obtain end-to-end statistics such as loss, latency, and throughput [9]. Then the trusted controller collects these statistics for further reasoning. Active probing techniques usually consume the larger bandwidth of networks, so it is important to design an appropriate probing strategy for active probing. Most researchers have focused on reducing the bandwidth utilization of probe packets [10-13]. On the other hand, a reasonable placement strategy of probe stations is also effective to reduce the count of the packet [14]. However, the previous study on active fault diagnosis ignores the information included in the latest detection, and the detective strategy on that may be obviously different with the change of network status.

(iii) Method based on the network syslog

With the development of the big-data techniques, fault diagnosis based on syslog data has been widely used. Most syslog-based methods locate faults by analyzing the alarm $\log$ sent with the network device [15]. Garshasbi et al. [16] proposed an end-to-end method to detect the network condition. Maggie et al. [17] also presented an end-to-end measurement to discover the correlation among network events by the regression model. Zhang et al. [18] analyzed the syslogs on switches to generate different syslog's templates, and then templates could be used to classify and locate faults. There are also lots of research on discovering alarm modes [19-21]. However, these proposed methods ignore the network uncertainty described in Section 1 , and most of these methods require static topology. In datacenter networks with dynamic topology, it is necessary to design a new flexible detection strategy.

As described above, although many techniques of fault diagnosis have been proposed, these methods still have their own inapplicability for datacenter networks. As opposed to most existing approaches, this research primarily focuses on problems of diagnosing faulty nodes quickly and accurately on the basis of information uncertainty, and reducing the communication cost effectively.

Xuan et al. [22] proposed a hierarchical fault diagnosis
(HFD) algorithm for datacenter networks. HFD divides the process of fault diagnosis into a logical layer and a correlation layer. In the logical layer, fault symptom of each node is determined by the convergence probability which is obtained with many times detection. Then, HFD identifies the faulty nodes through the cluster algorithm in the correlation layer. Although HFD reduces the detection flow, it still has the same problems of previously mentioned methods: (i) HFD ignores the indicated information that has been obtained during the last probe; (ii) it does not take full advantage of the dynamic topology of datacenter networks; (iii) it needs too many detection times to locate the faulty nodes.

Inspired by the end-to-end detective strategy of HFD, a proactive fault diagnosis algorithm, called heuristic breadth-first detection (HBFD), is proposed for fault diagnosis of the datacenter network. The HBFD algorithm tries to build a complete framework for fault diagnosis in uncertain conditions based on the Dempster-Shafer theory (DST) [23], and the heuristic detection strategy is also proposed to avoid using fault nodes to generate alarms for reducing network uncertainties.

In what follows, Section 2 gives an overview of the HBFD algorithm. In Section 3, details of each component of HBFD are described, and a theoretical analysis will be described to prove the effectiveness of HBFD. Simulation results are presented and discussed in Section 4.

\section{System overview}

An undirected graph $G=\langle N, L\rangle$ is used to model the datacenter network, which is composed of a set of nodes $N$ connected by a set of links $L$. As shown in Fig. 1, there are three examples of main topologies with higher connectivity [24]. Considering that the current technical level completely allows units in the network to communicate with each other, and then the node status can be obtained through the mutual testing between neighboring (adjacent) nodes. This detective method also saves diagnosis time and resource consumption by choosing appropriate detective paths and designing an effective algorithm. Thus, an appropriate spanning tree is designed, and then calculate the failure probability of each node through analyzing dial-test data based on the process of probabilistic assignment. Finally, identify the fault node after classifying the suspicious node set into the fault node set and the normal node set, based on a calculated threshold.

HBFD includes three functional components: dynamic spanning tree searching (DSTS), fault probability evaluation (FPE), and fault reasoning (FR). This section briefly describes the main idea of each component as shown in Fig. 2. Additional detail algorithms will be presented in Section 3. 


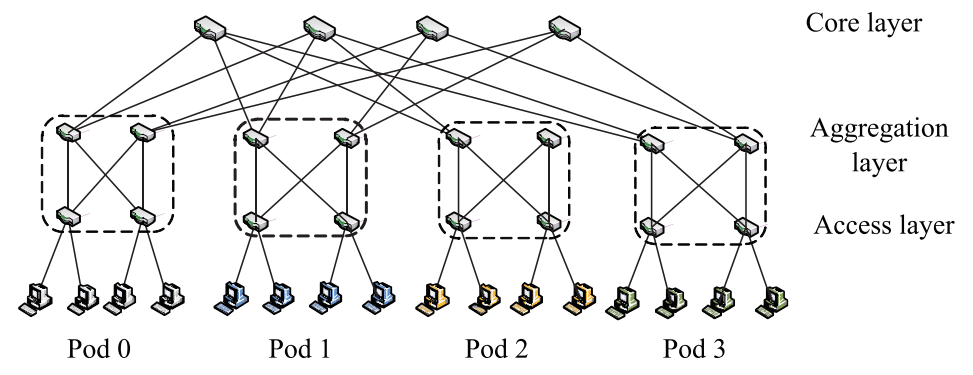

(a) Fat tree topology

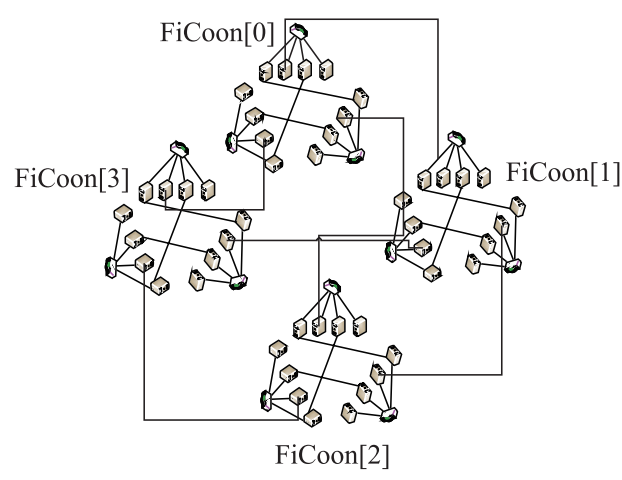

(b) FiCoon topology

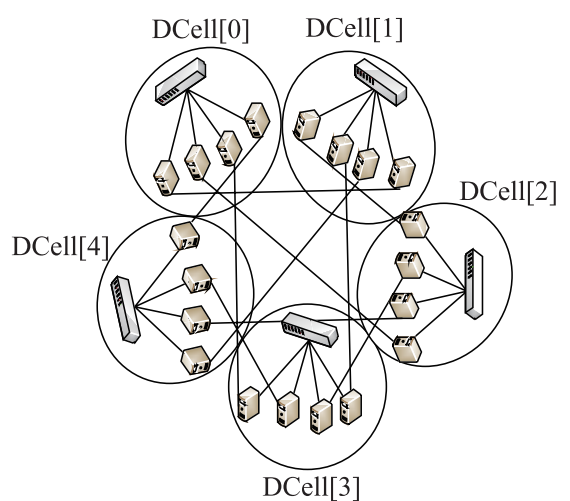

(c) DCell topology

Fig. 1 Three main datacenter network topologies

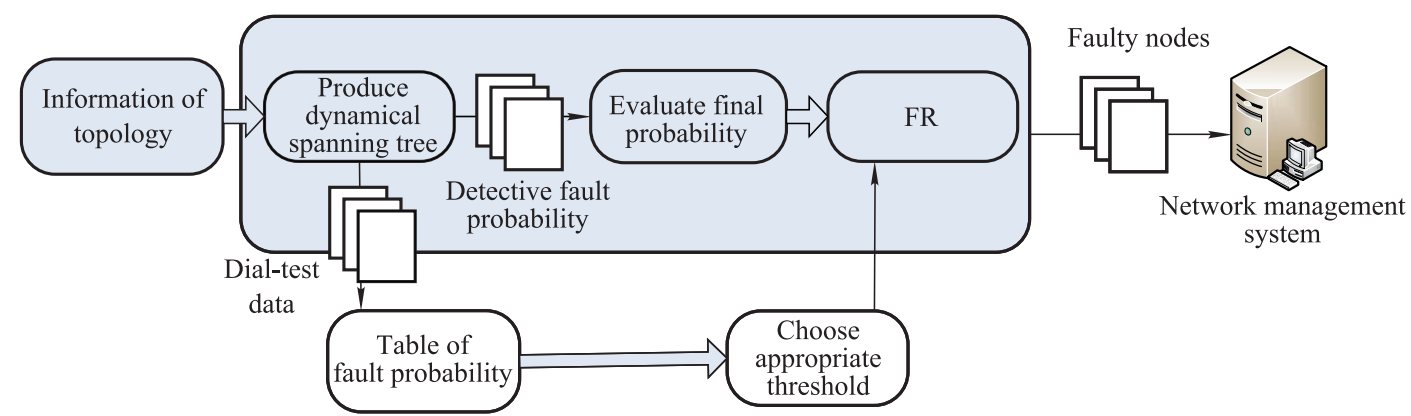

Fig. 2 System overview

Definition 1 Given a dynamical spanning tree, the tested status $s_{i}$ between any pair of adjacent nodes, also called a symptom, is composed of two indicator variables $s_{i}=\left\{r_{i j}, r_{j i}\right\}$, where $r_{i j}\left(r_{j i}\right)$ is the result obtained by the testing node $i(j)$ from the node $j(i)$. Here, $r_{i j}=0$ means that the node $i$ identifies the node $j$ as a normal node, and $r_{i j}=1$ implies the node $j$ is identified as being in a bad condition by the node $i$. The vector of $s_{i}$ is called the symptom matrix $\boldsymbol{S}$.

The component of DSTS can dynamically find a spanning tree based on the last diagnosis result. Then HBFD will avoid using the faulty node to test other suspicious nodes as much as possible and reduce the uncertainties of the probe result, e.g., when a faulty node $i$ tests other nodes, the symptom $s_{i}$ may indicate good conditions to the faulty node.
The FPE component quantifies fault probability of each suspicious node $n(n \in \mathbf{N})$. As described in Section 3, the fault probability of $n$ depends on not only its prior probability, i.e., a probability that a faulty node diagnoses correctly, but also the combination of diagnostic results from other nodes. FPE gets the detective fault probability based on the table of an m-function, and then calculates the final fault probability through the combination function, also called the decision function. The higher rate the final fault probability has, the more likely the node $n$ is faulty.

Finally, all fault probabilities of suspicious nodes are fed to the FR component. First, FR puts absolute faulty nodes (of which the fault probability equals 1 ) into the fault group. Then, FR chooses an appropriate threshold based on the $\mathrm{m}$-function table to classify suspicious nodes into two groups. At last, suspicious nodes can be divided into a rel- 
ative faulty group $F$ and a relative normal group $N F$ by choosing a classification threshold.

Considering the versatility of the HBFD algorithm, this research focuses on a network with arbitrary topology. No malicious nodes exist in the network and at least one trusted administrative controller can be used to collect the dial-test data and run the HBFD algorithm.

\section{Heuristic fault diagnosis}

In this section, the three components of HBFD will be presented in detail. Taking into account the dynamic traffic pattern in the current datacenter network and the versatility of our algorithm, our design completely removes the assumption of any particular network topology. This research also does not consider malicious nodes and link failures, and argues that it is feasible to deploy the HBFD algorithm in different datacenter network topologies, e.g., VL2, FiCoon, and DCell. Even though HBFD is a logical single entity, it can still be implemented in a distributed way by analyzing dial-test data distributed storage.

\subsection{Problem analysis}

Faults are network events that are the root cause of problems that occur in the network [25]. A node failure occurs when the device is not functioning for routing or forwarding traffic. The node failure may be caused by many factors such as a device being powered down for maintenance or crashing due to hardware errors, packet loss or time-out response when the network traffic is heavy. All of these failures may cause uncertain results of detection.

Proactive probing transmits one or more packets to nodes in the network for detecting the status of each node. According to the preparata metze chien (PMC) model definition, there are six different results for a group of intertest nodes: (i) the results of two normal nodes testing each other must be normal, e.g., $r_{i j}=0, r_{j i}=0$; (ii) when a normal node detects a failed node, the result must be failure, e.g., $r_{i j}=0, r_{j i}=1$; (iii)-(vi) the results from fault nodes are uncertain, e.g., $r=\{0,1\}$, no matter what the state of nodes under test is.

In this paper, the behavior shown by the failed node is defined as a symptom, e.g., $r=1$. Two simple examples will be described to illustrate the uncertainties caused by the failed node in the network as shown in Fig. 3.

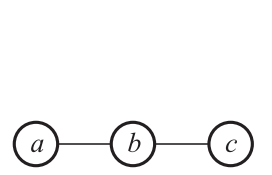

(a) Topology 1

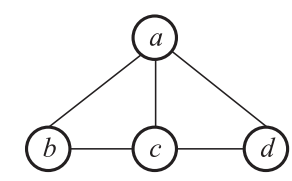

(b) Topology 2

Fig. 3 Fault detection graph
Example 1 In Fig. 3(a), $\{a, b, c\}$ are three nodes of the network, and we assume that $a$ and $c$ are two faulty nodes, and $b$ is in good condition. Then the probe result of $\{a, b\}$ may be one of the set: $\{(0,1),(1,1)\}$, and the result of $\{b, c\}$ may be one of the set: $\{(1,0),(1,1)\}$. When the symptom group is $\{(0,1),(1,0)\}$, we will consider that the node $b$ is in good condition; but when the symptom group is $\{(1,1),(1,1)\}$, we will consider that the node $b$ is in bad condition, and the remaining combinations are not sufficient to identify the condition of the node $b$.

Example 2 In Fig. 3(b), when the node $a$ is faulty, no matter what conditions of nodes $\{b, c, d\}$ are, the conditions of these four nodes will be uncertain.

Definition 2 A faulty node takes the correct detection result according to a given probability. This certain probability is defined as the priori probability $p$.

As a given priori probability $p$, for a faulty node $n_{i}$, when it probes a normal node, it gets the symptom of $r_{i j}=0$ with the probability $p$, and the symptom of $r_{i j}=1$ with the probability $1-p$.

Given a network, when the network status, e.g., connectivity, time latency and packet loss rate, is relatively well, the priori probability $p$ may be higher, and then the dialtest data will be more effective. The probing results may be completely accurate when $p=1$, but it exists only in an ideal case. Detailed analysis of $p$ belongs to the field of network performance assessment, and to different networks, it may need different expert experiences to determine.

The goal of fault diagnosis is to determine the status of each component, and then the fault diagnosis problem can be transformed as a decision problem under uncertain detection. Thus, the DST is introduced to handle the uncertain detection result.

The DST was proposed by Dempster and improved by Shafer, Dempster's student. The DST can represent and deal with uncertainties, and take a feature that satisfies the conditions, which is weaker than the Bayesian probability theory. For a given question, the DST defines the set of hypotheses $\Theta$ as the condition set of components. Let $2^{\Theta}=\{A \mid A \in \Theta\}$, the set of all subset of $\Theta$, be the recognition framework which represents all possible solutions to a problem, and there is only one correct answer $\left(A \in 2^{\Theta}\right)$ to the question. DST also assigns a belief probability by a function called basic belief assignment function (m-function):

$$
\left\{\begin{array}{l}
m\left(2^{\Theta}\right) \rightarrow[0,1] \\
m[\varnothing]=0 \\
\sum_{A \in 2^{\Theta}} m\left(2^{\Theta}\right)=1
\end{array} .\right.
$$

In the DST, different beliefs based on independent evidences from different sources, e.g., two anti-neighbor 
nodes, can be combined by the Dempster's combination rule. Suppose $m_{1}$ and $m_{2}$ are two irrelevant basic belief assignment functions, and share the same recognition framework $\Theta$, the combined probability can be calculated as follows:

$$
m_{12}(X)=\left(m_{1} \oplus m_{2}\right)(X)
$$

where $\oplus$ represents the combination method of $\mathrm{m}$ function.

To our problem, the evidence is the detective result (symptoms), and the network uncertainty is determined by the symptoms from faulty nodes. Thus, it has $\Theta=\{0,1\}$ for each component of the network. Designing a point-topoint fault diagnosis technique based on the DST mainly includes three steps.

(i) Define the frame of discernment for the given question, which also means the condition set of nodes in the network. HBFD obtains the set of symptoms by the component of DSTS.

(ii) Design an appropriate basic belief assignment function and a combination rule to assign belief probability based on the test result (symptom), and this component is designed in FPE.

(iii) Choose a reasonable category threshold based on the m-function table.

\subsection{DSTS}

To detect the node failure, the first thing HBFD needs to do is to find a spanning tree for a given network topology, such that nodes can probe each other with links in the spanning tree. As mentioned above, detection results of the fault node are uncertain even a priori probability is introduced to measure uncertainty, only once detection progress is insufficient to find all fault nodes.

As mentioned in Section 2, datacenter networks have high connectivity. Thus, for large-scale networks, using the breadth-first searching (BFS) algorithm to find a spanning tree can increase the speed of finding appropriate probing paths. BFS is also effective to avoid single diagnosis which causes inaccurate results as shown in Fig. 3. Based on BFS, a heuristic BFS (HBFS) algorithm is designed as shown in Algorithm 1, where $N$ is a set of nodes in the network, $N F$ is a set of normal nodes, and $F$ is a set of failure nodes.

\section{Algorithm 1 HBFS}

Step $1 F \leftarrow$ the last time detection result;

Step $2 F=\varnothing$, turn to Step 3; else, turn to Step 4;

Step 3 Find the breadth-first spanning tree by a classical algorithm;

Step $4 N F \leftarrow N-F$, use $N F$ as the initial searching nodes.
The HBFS algorithm dynamically uses the last probe result, which includes some absolute failure nodes, i.e., of which the fault probability is 1 , to find a new spanning tree that avoids using fault nodes to probe other nodes. Through reducing the uncertainty of probing result and communication times, the algorithm of HBFS effectively improves the accuracy and speed of detection.

\subsection{FPE}

For a given datacenter network, the result of 0 is defined as the good condition of a node, and the result of 1 as the bad condition. All detection results can be obtained by processing DSTS, and then the m-function of each result is calculated based on $p$.

The detective fault probability of each node can be gained based on the table of the m-function, but the condition of each node is determined by several nodes as shown in Section 3.1. Thus, combined with the DST, the combination function $\psi_{c}$, also called the decision function, is designed to calculate the faulty probability of each node.

$$
\left\{\begin{array}{l}
f\left(n_{j}\right)=\max \left\{e_{i j} \mid e_{i j} \in E\right\}\left(n_{j} \in N\right) \\
\psi_{c}\left(n_{j}\right)=\left\{\begin{array}{l}
1, \quad f\left(n_{j}\right)=1 \\
\min \left\{e_{i j} \mid e_{i j} \in E\right\}\left(n_{j} \in N\right), \quad f\left(n_{j}\right)<1
\end{array} .\right.
\end{array}\right.
$$

The unique probability of node failure can be evaluated based on $\psi_{c}$. Then, HBFD locates the faulty nodes through the component of FR.

\subsection{FR}

After the fault evaluation component, HBFD can get the final probability of each node. HBFD first puts the absolute faulty nodes, e.g., $\psi_{c}(\cdot)=1$, to the set of faulty nodes $F$, then chooses two appropriate thresholds based on the table of fault probability. To those four components in Table 1, conclusions can be derived as follows.

Table 1 m-function

\begin{tabular}{ccccc}
\hline Function & $r_{i j}$ & $r_{j i}$ & $\mathrm{DE}(i)$ & $\mathrm{DE}(j)$ \\
\hline$m_{1}$ & 0 & 0 & $(1-p)^{2}$ & $(1-p)^{2}$ \\
$m_{2}$ & 1 & 0 & $p(1-p)$ & 1 \\
$m_{3}$ & 0 & 1 & 1 & $p(1-p)$ \\
$m_{4}$ & 1 & 1 & $\frac{1}{2}+\frac{p^{2}}{2}$ & $\frac{1}{2}+\frac{p^{2}}{2}$ \\
\hline
\end{tabular}

(i) The result of $m_{1}$ represents the inter-test nodes are in the same condition. When the priori probability $p \geqslant 0.3$, fault probabilities of those two nodes are lower than 0.5 . Then we put them to the normal node set to the next detection. When the priori probability $p<0.3$, fault probabilities are higher than 0.5 . It may cause that the decision function assigns a high probability to the normal inter-test 
nodes, which means that there are malicious nodes providing false information to disturb the process of fault diagnosis. Thus, we assume that there are malicious nodes in the network when $p<0.3$, but we do not consider this condition.

(ii) Components of $m_{2}$ and $m_{3}$ indicate that there is an absolute fault node, e.g., detective fault probability is 1 , between inter-detect nodes, and the fault probability of another one is low no matter what $p$ value is, so we consider another node as a normal node.

(iii) The inter-test nodes of $m_{4}$ have at least one node failure, and probabilities are always higher than 0.5 ; we put both of them to the suspicious node set to further detection.

In summary, the threshold of classification is set to 0.5 and it also sets $p \geqslant 0.3$. Thus, nodes with $\psi_{c}>0.5$ will be placed to the set of faulty nodes, and then be assigned to the set $F$ in Algorithm 1 to avoid faulty nodes detecting other units in the network.

\subsection{Theoretical analysis}

In this section, theoretical analysis is described to prove that fault diagnosis rate and detection times will be improved as reducing the number of failed nodes in the detective set.

As shown in Fig. 3(b), nodes $b, c$, and $d$ are used to detect the condition of the node $a$. Under the condition that $a$ is a faulty node, there are two different results of $a$ 's detective result.

Result 1 If $b, c$, and $d$ are all faulty nodes, possible symptoms and corresponding probabilities are shown in bellow:

$$
\left\{\begin{array}{l}
P(s=(0,0))=(1-p)^{2} \\
P(s=(1,0))=p(1-p) \\
P(s=(0,1))=p(1-p) \\
P(s=(1,1))=p^{2}
\end{array} .\right.
$$

It is obvious that

$$
\begin{gathered}
P(s=(1,0))+P(s=(1,1))<P(s=(0,0))+ \\
P(s=(0,1)), \quad p<0.5 .
\end{gathered}
$$

Thus, it takes a high probability to put the node $a$ to the normal set and starts a new round of detection to identify faulty nodes when a large-scale failure occurs in the network.

Result 2 There is at least one normal node among $b, c$ and $d$. Then one of the detection results of $(1,0)$ and $(1,1)$ will definitely appear, and it also has

$$
\left\{\begin{array}{l}
P(s=(1,1))=(1-p)+2 p^{2} \\
P(s=(1,0))=p+2 p(1-p) \\
P(s=(0,0))=2(1-p)^{2} \\
P(s=(0,1))=2 p(1-p)
\end{array} .\right.
$$

It is obvious that

$$
\begin{gathered}
P(s=(1,0))+P(s=(1,1))> \\
P(s=(0,0))+P(0,1) .
\end{gathered}
$$

Thus, it takes a high probability to put the node $a$ to the set of faulty nodes.

In summary, it has been proved that the fault diagnosis rate and detection times will be improved as reducing the number of failed nodes in the detective set. It also means that the HBFD is effective to identify faulty nodes even if failure symptoms are uncertain.

\section{Simulation results}

As proactive point-to-point fault diagnosis is based on dialtest data in datacenter networks, HBFD can be flexibly deployed and integrated with the existing routing protocols, in order to improve fault diagnosis accuracy and reduce their monitoring cost. Evaluation metrics, simulation results, and performances of the proposed algorithm are presented in this section.

\subsection{Evaluation metrics}

Evaluation metrics include detection numbers (DNs), fault granularity (FG), correct diagnosis rate (CDR), and false diagnosis rate (FDR).

DNs are used to measure the communication time used in fault diagnosis to get dial-test data. An algorithm with a lower value of $\mathrm{DN}$ is better. Let $P F$ be the practical faulty nodes. $\mathrm{FG}$ is the ratio between $|P F|$ and the network size $|N|$.

$$
\mathrm{FG}=\frac{|P F|}{|N|}
$$

CDR and FDR are two evaluation metrics to measure the accuracy of the fault diagnosis algorithm written as

$$
\begin{aligned}
& \mathrm{CDR}=\frac{|F \cap P F|}{|P F|} \\
& \mathrm{FDR}=\frac{|P F-F|}{|F|} .
\end{aligned}
$$

\subsection{Results}

Considering the applicability of the HBFD algorithm, we generate network topology and choose failure nodes randomly. Our simulation results are shown in Figs. 4-6. The performance of our algorithm will be analyzed from three aspects. 
QI Xiaogang et al.: Fault diagnosis based on dial-test data in datacenter networks

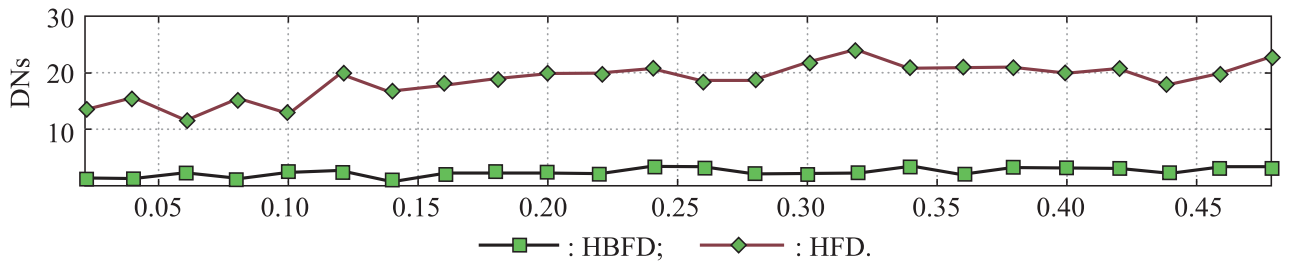

$\mathrm{FG} / \%$
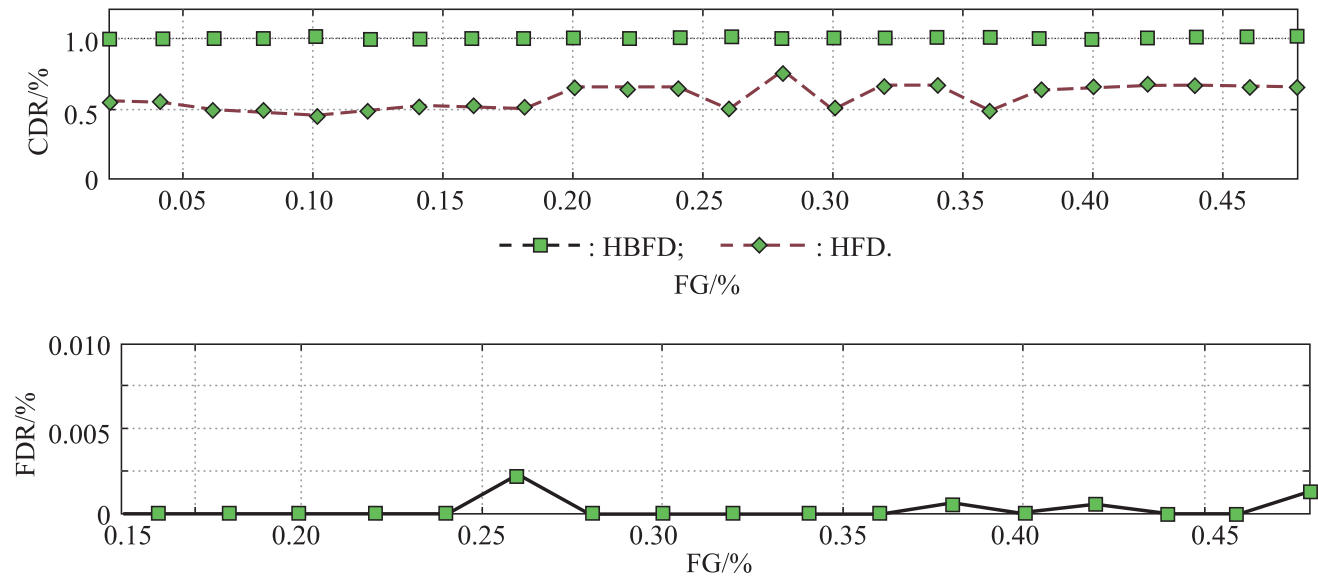

Fig. 4 Different evaluation metrics of the results from HBFD and HFD

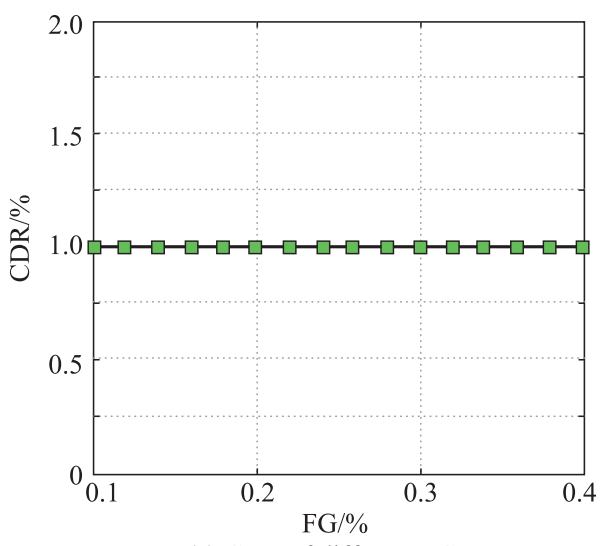

(a) CDR of different FGs

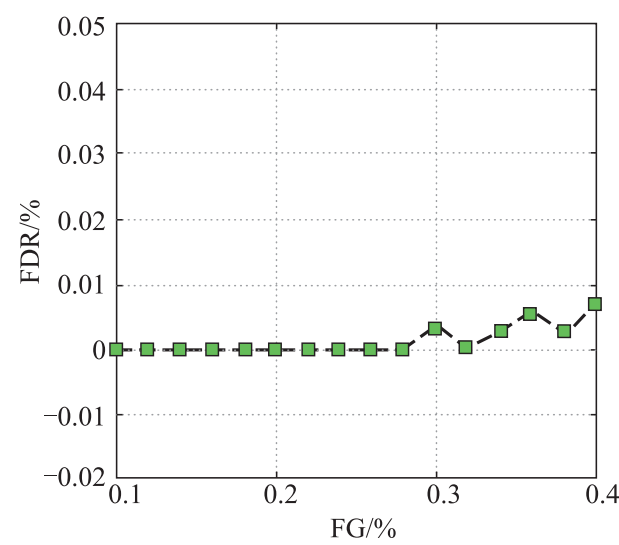

(b) FDR of different FGs

Fig. 5 Impact of FG

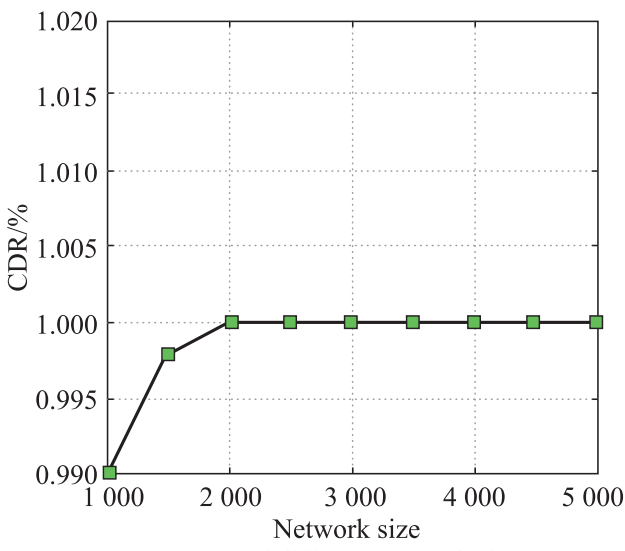

(a) CDR of different network sizes

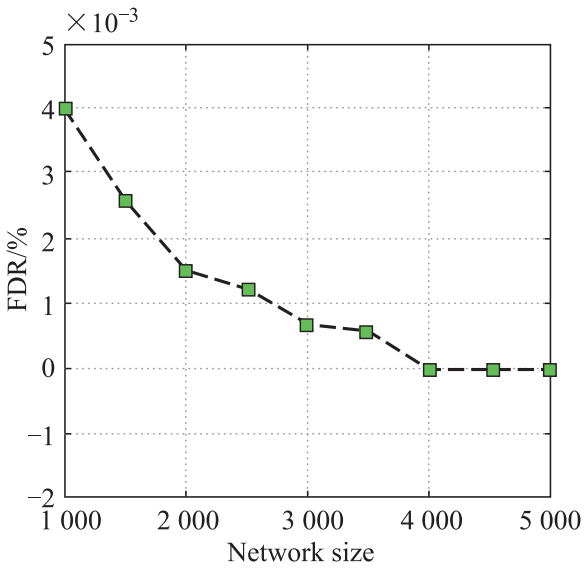

(b) FDR of different network sizes

Fig. 6 Impact of network size 


\section{(i) Advantages of HBFD}

The advantages of the HBFD algorithm compared to the HFD algorithm are shown in Fig. 4. The network size, the value of the priori probability and the range of detection granularity are set by $5000,0.3$ and $[0.05,0.5]$, respectively. It is straightforward to find that metrics of DNs and CDR have been well improved by the HBFD algorithm. It also means that, avoiding using faulty nodes to detect other nodes is effective to increase diagnosis accuracy, and reduce the invalid communication effectively. However, there is a drawback that HBFD may cause a very low FDR as shown in Fig. 4. Analysis of the reason is described as follows.

\section{(ii) The impact of FG}

As shown in Fig. 5, FG is set in the range [0.1, 0.4]. The diagnosis accuracy is still very stable. However, as the FG rises, the FDR has a slight increase. This is because that higher FG indicates more failed nodes in the network. HBFS cannot find a dynamic spanning tree without faulty nodes. As described in Table 1, the forth symptom appears with a higher probability. Thus, there are a few normal nodes identified as failure nodes.

(iii) The impact of network size

This research gives different network sizes ranging in [1 000, 5 000] and the same FG 0.5 as shown in Fig. 6. It is obvious that the CDR is stable in different scales of networks, and the FDR is decreasing with the increase of the network size. The reason is that, under the same FG, the expansion of the network size will cause the number of normal nodes in the network to rise. As described is Section 3.5 , the probability of wrong diagnosis will decrease with the addition of normal nodes in the detective set. These results implicate that HBFD is robust in different scales of networks and takes a lower FDR.

\section{Conclusions}

In this paper, a point-to-point fault diagnosis algorithm is proposed to detect and locate the most likely faulty nodes based on dial-test data. Compared to the HFD algorithm, HBFD has better performance in terms of both detection numbers and diagnosis accuracy. HBFD is also robust in different scales of networks, but it has a minor false positive rate when the FG is relatively higher or the network condition is poor, e.g. higher rate of packet loss, congestion or malicious nodes. Thus, in our future work, how to eliminate the false positive rate and identify the malicious nodes is the focus of research. We also plan to target on more practical scenarios and apply the HBFD algorithm to more specific topologies, and combine it with different transport protocols.

\section{References}

[1] GUO C, YUAN L H, XIANG D, et al. Pingmesh: a largescale system for data center network latency measurement and analysis. Proc. of the ACM Conference on Special Interest Group on Data Communication, 2015, 45(4): 139- 152.

[2] CHEN T, GAO X F, CHEN G H. The features, hardware, and architectures of data center networks: a survey. Journal of Parallel \& Distributed Computing, 2016, 96(C): 45 - 74.

[3] WANG B, WEI W, DINH H, et al. Fault localization using passive end-to-end measurements and sequential testing for wireless sensor networks. IEEE Trans. on Mobile Computing, 2012, 11(3): 439-452.

[4] NIE J W, MA H D, MO L F. Passive diagnosis for WSNs using data traces. Proc. of the IEEE International Conference on Distributed Computing in Sensor Systems, 2012: 273-280.

[5] LEI X P, WANG C Q. Ping command under the multimedia passive network fault diagnosis methods. Computer Simulation, 2017, 34(8): 369-372. (in Chinese)

[6] MEHMOOD A, ALRAJEH N, MUKHERJEE M, et al. A survey on proactive, active and passive fault diagnosis protocols for WSNs: network operation perspective. Sensors, 2018, 18(6): $1787-1807$.

[7] GU M M, HAO R X, LIU J B. The pessimistic diagnosability of data center networks. Information Processing Letters, 2018, 134: $52-56$

[8] LIANG J R, ZHOU N, YUN L. T/k-fault diagnosis algorithm of $n$-dimensional hypercube network based on the MM* model. Journal of Systems Engineering and Electronics, 2018, 29(1): $216-222$

[9] ZHANG X, ZHOU F F, ZHU X Y, et al. DFL: secure and practical fault localization for datacenter networks. IEEE/ACM Trans. on Networking, 2014, 22(4): 1218-1231.

[10] LU L, XU Z G, WANG W H, et al. A new fault detection method for computer networks. Reliability Engineering \& System Safety, 2013, 114: 45-51.

[11] BELLALA G, STANLEY J, BHANANAI S K, et al. A rankbased approach to active diagnosis. IEEE Trans. on Pattern Analysis \& Machine Intelligence, 2013, 35(9): 2078-2090.

[12] ALEKSEEV D, SAYENKO V. Proactive fault detection in computer networks. Proc. of the 1st International ScientificPractical Conference Problems of Infocommunications Science and Technology, 2014: 90-91.

[13] WANG J X, QI H, LI K Q, et al. Real-time link fault detection as a service for datacenter network. Journal of Computer Research \& Development, 2018, 55(4): 704-716. (in Chinese)

[14] NATU M, SETHI A S. Probe station placement for fault diagnosis. Proc. of the IEEE Global Telecommunications Conference, 2007: 113-117.

[15] GILL P, JAIN N, NAGAPPAN N. Understanding network failures in data centers: measurement, analysis, and implications. ACM SIGCOMM Computer Communication Review, 2011, 41(4): $350-361$.

[16] GAISHASBI M S, JAMALI S. A new fault detection method using end-to-end data and sequential testing for computer networks. International Journal of Information Technology \& Computer Science, 2013, 6(1): 93-100.

[17] CHENG M, WU W B. Data analytics for fault localization in complex networks. IEEE Internet of Things Journal, 2017, 3(5): $701-708$.

[18] ZHANG S L, MENG W B, BU J H, et al. Syslog processing for switch failure diagnosis and prediction in datacenter networks. Proc. of the IEEE/ACM 25th International Symposium on Quality of Service, 2017: 1-10.

[19] MYSORE R N, MAHAJAN R, VAHDAT A, et al. Gestalt: 
fast, unified fault localization for networked systems. Proc. of the Usenix Conference on Usenix Technical Conference, 2014: $1-12$.

[20] SEIPEL D, NEUBECK P, STEFAN K, et al. Mining complex event patterns in computer networks. APPICE A, CECI $\mathrm{M}$, LOGLISCI C, et al. Ed. New frontiers in mining complex patterns. Heidelberg Berlin: Springer, 2013: 33-48.

[21] WANG J T, HE C F, LIU Y J, et al. Efficient alarm behavior analytics for telecom networks. Information Sciences, 2017, 402: $1-14$.

[22] XUAN H N, ZHANG R C, ZUO M, et al. A hierarchical fault diagnosis algorithm for data center networks. Acta Electronica Sinica, 2014, 42(12): 2536-2542. (in Chinese)

[23] YAGER R R. On the dempster-shafer framework and new combination rules. Information Sciences, 1987, 41(2): $93-$ 137.

[24] WAN H T. Research on data center network topology. Computer Knowledge and Technology: Academic Exchange, 2016, 12(21): $43-45$.

[25] DUSIA A, SETHI A S. Recent advances in fault localization in computer networks. IEEE Communications Survey \& Tutorials, 2016, 18(4): 3030-3051.

\section{Biographies}

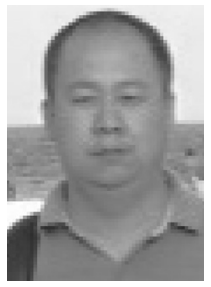

QI Xiaogang was born in 1973. He is a professor and Ph.D. supervisor in School of Mathematics and Statistics of Xidian University. He received his Ph.D. degree in applied mathematics from Xidian University in 2005 , and joined as a faculty member in the same university in 2002, and an associate professor in 2006. From September 2012 to August 2013, he was a visiting scholar in School of Electrical, Computer and Energy Engineering of Arizona State University, Tempe, AZ. His current research interests include system modeling and simulation, resource management and schedule, performance evaluation and optimization algorithm design, and fault diagnosis in various networks.

E-mail: xgqi@xidian.edu.cn

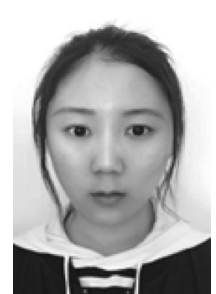

WANG Bingchun was born in 1997. She received her B.S. degree from Guilin University of Technology, China, in 2016. She is now a graduate student at Xidian University. Her current research interests include data analysis and fault diagnosis of networks.

E-mail: 1066955863@qq.com

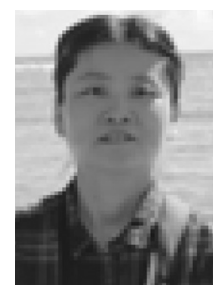

LIU Lifang was born in 1972. She received her $\mathrm{Ph} . \mathrm{D}$. degree in computer application from Xidian University in 2006, and became an associate professor in the same year. Since 2015, she has become a professor in School of Computer Science and Technology of Xidian University. Her current research interests include computer network, algorithm design and analysis, data processing and intelligent calculation.

E-mail: 1fliu@xidian.edu.cn 